\title{
Interplay between Coulomb blockade and Josephson effect in a topological superconductor-quantum dot device
}

\author{
Yu-Li Let* \\ Department of Physics, National Changhua University of Education, Changhua, Taiwan, R.O.C. \\ Yu-Wen Le丹丹 \\ Department of Applied Physics, Tunghai University, Taichung, Taiwan, R.O.C.
}

(Dated: October 11, 2018)

\begin{abstract}
We study the behavior of a topological Josephson junction in which two topological superconductors are coupled through a quantum dot. We focus on the case with the bulk superconducting gap being the largest energy scale. Two parameter regimes are investigated: a weak tunneling between the dot and the superconductors, with the dot near its charge degeneracy point, and a strong tunneling regime in which the transmission between the dot and the superconductors is nearly perfect. We show that in the former situation, the Andreev spectrum for each sector with fixed fermion parity consists of only two levels, which gives rise to the nontrivial current-phase relation. Moreover, we study the Rabi oscillation between the two levels and indicates that the corresponding frequency is a $4 \pi$-periodic function of the phase difference between the two superconductors, which is immune to the quasiparticle poisoning. In the latter case, we find that the Coulomb charging energy enhances the effect of backscattering at the interfaces between the dot and the superconductors. Both the temperature and the gate-voltage dependence of the critical Josephson current are examined.
\end{abstract}

PACS numbers: $74.50 .+\mathrm{r} 73.63 . \mathrm{Kv} 74.45 .+\mathrm{c}$

\section{INTRODUCTION}

Over the past few years, the Majorana bound states (MBSs) in topological superconductors (TSCs) have attracted a lot of attention in the condensed matter research community $\underline{\underline{1}} \underline{\underline{4}}$. These exotic objects are basically the zero-energy quasiparticles which are the equalamplitude superposition of particles and holes in TSCs. One surprising characteristic of these MBSs is that they obey non-Abelian braiding statistics ${ }^{5}$. Furthermore, a widely separated pair of MBSs forms a nonlocal fermionic state which is immune to local sources of decoherence. Both features provide a potential for future applications in quantum computing and quantum information processes $^{\underline{6}-\underline{8}}$. Up to now, there are several candidate systems that are promised to realize these MBSs. Typically, s-wave superconductors are put in proximity to other materials with strong spin-orbital coupling, in the presence or absence of external magnetic fields $\underline{1-3}$. There have already been several recent experiments investigating these kinds of systems ${ }^{9}-12$.

One way to reveal the existence of the MBSs is through the $4 \pi$ (or fractional) Josephson effect in a topological Josephson junction ${ }^{6}$ 13-15, where the DC Josephson current is a $4 \pi$-periodic function of the superconducting (SC) phase difference between the two TSCs. This doubling of the period is tightly related to these zeroenergy MBSs. Roughly speaking, the existence of zeroenergy MBSs allow the coherent single-particle tunneling between TSCs. Since the electron carries half of the SC phase, this results in the doubling of the period. However, in real experimental situations, there are many subtle effects, such as disorder, multiple bands, quasiparticle poisoning, Coulomb charging energy for SC is- lands etc., which have to be taken into account $\stackrel{4,15}{-19}$. Therefore, it is extremely important to understand how this $4 \pi$ Josephson effect survives these complications.

There are several recent attempts in this research direction. For example, by coupling the topological Josephson junction to an external normal-metal probe, the dynamics of the fermion-parity switch through the ejection of quasiparticles via the probe is analyzed in Ref. 20. In Ref. 21, the effects of electromagnetic environments and an additional quantum dot (QD) on the charge transport between the TSC and a normal-metal lead are considered. Interestingly, in the latter case, it is claimed that when two TSCs are coupled via a $\mathrm{QD}$, the resulting system is equivalent to a resonant-level model at low energy from which a Coulomb oscillation in the conductance follows in both the weak and the strong tunneling regime. It is not clear how the Josephson current, which is supposed to exist in the absence of the QD, is affected by the presence of the QD.

The interesting situation where the TSC is coupled to a metallic lead through a QD has already been studied $^{22-25}$. There, the point is to analyze the competition between the Andreev reflection and the Coulomb blockade/Kondo effect. In the present work, we would like to study the effects of Coulomb blockade, introduced by a QD, on the DC Josephson current. The TSC itself forms a ring with a gap, and the ring is threaded by a magnetic flux $\Phi_{B}$ which determines the SC phase difference $\phi$ between the two ends of the TSC. The two ends of the TSC is coupled through a QD to form a TSC-QDTSC junction. A schematic setup is shown in Fig. 1. We focus on the case where the bulk SC gap is the largest energy scale. In the weak tunneling limit, we consider the case where the dot is near the charge degeneracy point. 


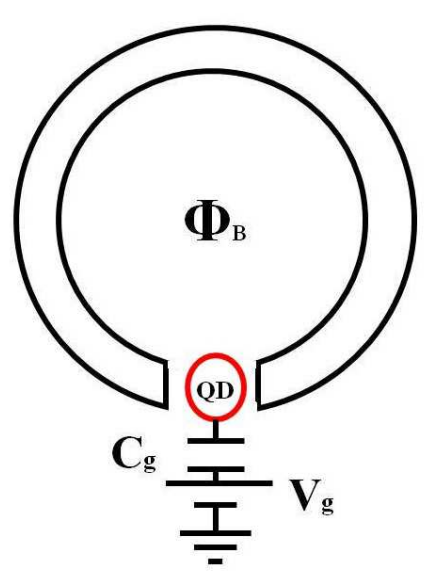

FIG. 1: (Color online) A schematic setup of the TSC-QDTSC junction. The TSC itself forms a ring threaded by a magnetic flux $\Phi_{B}$. The energy levels in the dot can be tuned by a capacitively coupled gate voltage $V_{g}$. The couplings between the QD and the TSC can be adjusted by additional gate voltages, which are not shown in the figure.

This is because in this case the Andreev spectrum, arising from the hybridization between the MBSs at the ends of the TSC and the charge degrees of freedom of the QD, shows a simple structure, i.e. two Andreev levels for each sector with fixed fermion parity. This results in a nontrivial current-phase relation with a $4 \pi$ periodicity. This simple structure of the Andreev spectrum and its $4 \pi$ dependence on $\phi$ can be revealed as sharp peaks in the spectral function of the dot, which could be measured by $\mathrm{STM}^{26}$ or microwave-optical experiments 27 . Moreover, by changing the flux through the ring abruptly, the Josephson current exhibits the behavior of Rabi oscillation with the frequency being a $4 \pi$-periodic function of $\phi$. This $\phi$ dependence of the Rabi frequency is immune to quasiparticle poisoning. Both features, the $4 \pi$ dependence of the peaks in the spectral function of the dot and the Rabi frequency, can be viewed as the hallmark for the presence of the MBSs. In the strong tunneling regime where the transmission between the QD and the SC electrodes is nearly perfect, the current-phase relation is the usual one, i.e. $I=I_{c} \sin (\phi / 2)$, provided that the fermion parity is conserved. We calculate the temperature and the gate-voltage dependence of the critical current $I_{c}$ by assuming that the temperature is much larger than the average level spacing in the dot. Due to the Coulomb charging energy of the dot, the backscattering at the interfaces between the QD and the SC electrodes is enhanced, which is revealed in the temperature dependence of $I_{c}$. Our main results are summarized in Figs. 2 and 3 ,

The rest of the paper is organized as follows. We present our analysis on the Andreev spectrum and the DC Josephson current in the weak and the strong tunneling regimes in Sec. II and III. respectively. The last section is devoted to a conclusive discussion.

\section{THE WEAK TUNNELING REGIME}

\section{A. The model}

We first consider the weak tunneling regime, i.e. $G_{l / r} \ll e^{2} / h$, where $G_{l / r}$ denotes the tunneling conductance for the left/right contact. At the energy scale much lower than the bulk SC gap $\Delta_{0}$, the system can be described by the Hamiltonian $H=H_{D}+H_{T}$, where

$$
H_{D}=\sum_{s} \epsilon_{s} d_{s}^{\dagger} d_{s}+E_{c}\left(\hat{N}-N_{g}\right)^{2}
$$

is the Hamiltonian of the dot with $E_{c}=e^{2} /(2 C)$ and

$$
H_{T}=\sum_{s}\left(\Gamma_{r s} e^{-i \chi_{r} / 2} \gamma_{r} d_{s}-i \Gamma_{l s} e^{-i \chi_{l} / 2} \gamma_{l} d_{s}+\text { H.c. }\right) \text {, }
$$

describes the tunneling between the two ends of the TSC and the QD, with the real tunneling amplitudes $\Gamma_{l s}$ and $\Gamma_{r s}$. We shall assume that $E_{c},\left|\Gamma_{l(r) s}\right| \ll \Delta_{0}$. Here $\gamma_{l}$ and $\gamma_{r}$, obeying the algebra $\gamma_{l}^{2}=1=\gamma_{r}^{2}$ and $\left\{\gamma_{l}, \gamma_{r}\right\}=0$, denote the two Majorana fermions at the ends of the left and the right SC electrodes, $\chi_{l}$ and $\chi_{r}$, obeying the constraint $\chi_{l}-\chi_{r}=2 e \Phi_{B} \equiv \phi$, are the SC phases of the left and the right $\mathrm{SC}$ electrodes, $\epsilon_{s}$ denotes the single-particle energy of the dot with the complete set of good quantum numbers $s$, the constant $N_{g}$ is proportional to the gate voltage $V_{g}$, the operators $d_{s}$ and $d_{s}^{\dagger}$ obey the canonical anticommutation relations, and the Hermitian operator $\hat{N}$, having integer eigenvalues, satisfies the commutation relations $\left[\hat{N}, d_{s}\right]=-d_{s}$.

Let $|N\rangle$ be the eigenstate of the operator $\hat{N}$ with eigenvalues $N=0, \pm 1, \pm 2, \cdots$. Following Ref. 28, for large charging energy, i.e. $E_{c} \gg\left|\Gamma_{l(r) s}\right|$, only two charge states $\left|N_{0}\right\rangle$ and $\left|N_{0}+1\right\rangle$ give dominant contributions to the low-energy properties near the degeneracy point $N_{g}=N_{0}+1 / 2$, where $N_{0}$ is some integer. To a good approximation, we can truncate the Hilbert space keeping only these two states. Within this truncated space, one may introduce a set of fermion operators $f$ and $f^{\dagger}$ defined by $f\left|N_{0}\right\rangle=0$ and $f^{\dagger}\left|N_{0}\right\rangle=\left|N_{0}+1\right\rangle$. Moreover, they obey the canonical anticommutation relations. Then, we have $\hat{N}-N_{0}=f^{\dagger} f$, and $H_{D}$ becomes

$$
H_{D}=\epsilon_{d} f^{\dagger} f
$$

up to a constant term, where $\epsilon_{d}$ accounts for the energy difference between the states $\left|N_{0}+1\right\rangle$ and $\left|N_{0}\right\rangle$, and can be written as $\epsilon_{d}=\epsilon_{0}-2 E_{c}\left(N_{g}-N_{0}-1 / 2\right)$. Here the constant $\epsilon_{0} \equiv\left\langle N_{0}+1\left|\sum_{s} \epsilon_{s} d_{s}^{\dagger} d_{s}\right| N_{0}+1\right\rangle-$ $\left\langle N_{0}\left|\sum_{s} \epsilon_{s} d_{s}^{\dagger} d_{s}\right| N_{0}\right\rangle$ is independent of $N_{g}-N_{0}$. On the other hand, $H_{T}$ can be written as

$$
H_{T}=t_{r} e^{-i \chi_{r} / 2} \gamma_{r} f-i t_{l} e^{-i \chi_{l} / 2} \gamma_{l} f+\text { H.c. },
$$


where $t_{l(r)} \equiv \sum_{s} \Gamma_{l(r) s}\left\langle N_{0}\left|d_{s}\right| N_{0}+1\right\rangle$. Without loss of generality, we may set $t_{l / r}>0$.

We see that near the degenerate point, the Hamiltonian $H=H_{D}+H_{T}$ is quadratic in the fermion operators $f$ and $\gamma_{l / r}$, and thus the whole problem can be solved exactly. To proceed, we introduce another set of fermion operators: $a=\left(\gamma_{l}+i \gamma_{r}\right) / 2$ and $a^{\dagger}=$ $\left(\gamma_{l}-i \gamma_{r}\right) / 2$, which obey the canonical anticommutation relations. Then, we may choose the set of orthonormalized states $\{|0,0\rangle,|1,1\rangle,|0,1\rangle,|1,0\rangle\}$ as the basis of the truncated Hilbert space, where the state $|m, n\rangle$ contains $m f$-fermions and $n a$-fermions. In terms of this choice of the basis, the Hamiltonian $H$ can be written as

$$
H=\left[\begin{array}{cc}
h_{e} & 0 \\
0 & h_{o}
\end{array}\right]
$$

where

$$
\begin{aligned}
& h_{e}=\left[\begin{array}{cc}
0 & -i\left(\Delta_{l}+\Delta_{r}\right) \\
i\left(\Delta_{l}+\Delta_{r}\right)^{*} & \epsilon_{d}
\end{array}\right], \\
& h_{o}=\left[\begin{array}{cc}
0 & -i\left(\Delta_{l}-\Delta_{r}\right) \\
i\left(\Delta_{l}-\Delta_{r}\right)^{*} & \epsilon_{d}
\end{array}\right],
\end{aligned}
$$

with $\Delta_{l / r} \equiv t_{l / r} e^{-i \chi_{l / r} / 2}$. We see that $H$ is block diagonal due to the fermion-parity symmetry of $H$. The Hermitian matrices $h_{e}$ and $h_{o}$ describe the dynamics of the states in the subspaces with even and odd fermion parity, respectively.

\section{B. The Andreev spectrum and the DC Josephson current}

We first study the Andreev spectrum and calculate the resulting DC Josephson current. The energy spectrum of $h_{e}$ is determined by its eigenvalues, which are given by

$$
E_{ \pm}(\phi)=\frac{1}{2}\left(\epsilon_{d} \pm \sqrt{\epsilon_{d}^{2}+4\left|\Delta_{l}+\Delta_{r}\right|^{2}}\right)
$$

with the corresponding eigenstates denoted by $| \pm ; \phi\rangle$, where $\left|\Delta_{l}+\Delta_{r}\right|^{2}=t_{l}^{2}+t_{r}^{2}+2 t_{l} t_{r} \cos (\phi / 2)$. The Andreev spectrum will exhibit sharp peaks in the spectral function of the dot, which is a $4 \pi$-periodic function of $\phi$. This structure could be measured by $\mathrm{STM}^{26}$ or microwaveoptical experiments 27 .

In terms of the formula $I_{e}=-2 e \partial F_{e} / \partial \phi$, where $F_{e}$ is the free energy of the junction with even fermion parity at finite temperature $T$, we get the DC Josephson current

$$
I_{e}(\phi)=I_{e 0}(\phi) \tanh \left[\frac{E_{+}(\phi)-E_{-}(\phi)}{2 T}\right],
$$

where

$$
I_{e 0}(\phi)=\frac{2 e t_{l} t_{r} \sin (\phi / 2)}{\sqrt{\epsilon_{d}^{2}+4\left|\Delta_{l}+\Delta_{r}\right|^{2}}}
$$
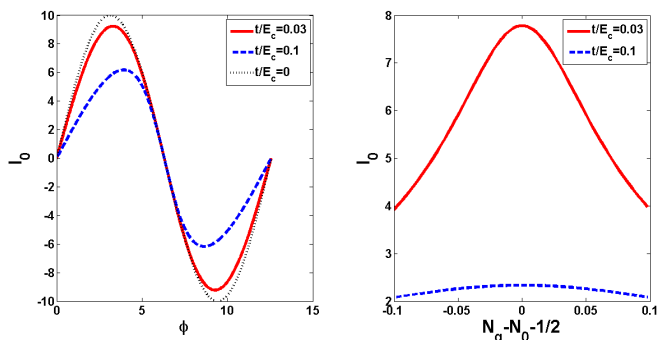

FIG. 2: (Color online) The DC Josephson current $I_{0}$ at $T=0$ for the TSC-QD-TSC junction in the weak-tunneling regime with $N_{g} \approx N_{0}+1 / 2$. We set $t_{l}=t_{r}=t, \epsilon_{0}=0$, and measure $I_{0}$ in units of $e t^{2} / E_{c}$. Only the results for the sector with even fermion parity are shown here. Left: the current-phase relation at $N_{g}-N_{0}-1 / 2= \pm 0.1$ for $t / E_{c}=0.03$ (solid line) and $t / E_{c}=0.1$ (dashed line). We also plot $I_{0}$ in the limit $t / E_{c} \rightarrow 0$ to show the deviation from the one $I_{0} \propto \sin (\phi / 2)$. Right: $I_{0}$ versus $N_{g}-N_{0}-1 / 2$ at $\phi=0.3 \pi$ for $t / E_{c}=0.03$ (solid line) and $t / E_{c}=0.1$ (dashed line).

is the DC Josephson current at $T=0$. We see that $I_{e}$ is a $4 \pi$-periodic function of $\phi$, a characteristic of the topological Josephson junction. In contrast with the short topological Josephson junction in which the DC Josephson current is independent of $T$ when $T \ll \Delta_{0} \underline{13}, I_{e}$ is a function of $T$ here.

For the subspace with odd fermion parity, the Andreev spectrum is given by

$$
\tilde{E}_{ \pm}(\phi)=\frac{1}{2}\left(\epsilon_{d} \pm \sqrt{\epsilon_{d}^{2}+4\left|\Delta_{l}-\Delta_{r}\right|^{2}}\right),
$$

where $\left|\Delta_{l}-\Delta_{r}\right|^{2}=t_{l}^{2}+t_{r}^{2}-2 t_{l} t_{r} \cos (\phi / 2)$. Hence, the DC Josephson current is of the form

$$
I_{o}(\phi)=I_{o 0}(\phi) \tanh \left[\frac{\tilde{E}_{+}(\phi)-\tilde{E}_{-}(\phi)}{2 T}\right],
$$

where

$$
I_{o 0}(\phi)=-\frac{2 e t_{l} t_{r} \sin (\phi / 2)}{\sqrt{\epsilon_{d}^{2}+4\left|\Delta_{l}-\Delta_{r}\right|^{2}}}
$$

is the DC Josephson current at $T=0$.

A few comments on the above results are in order. First of all, due to the presence of more than a single Andreev level for each sector with fixed fermion parity, the current-phase relation at $T=0$ deviates from the usual one proportional to $\sin (\phi / 2)$. Next, for given $\phi, I_{e 0}$ and $I_{o 0}$ have opposite signs, which is similar to the short topological Josephson junction ${ }^{13,15}$. Finally, the Andreev spectra for the sectors with even and odd fermion parity are related to each other through the relation: $E_{ \pm}(\phi \pm 2 \pi)=E_{ \pm}(\phi)$. Moreover, they cross each other at $\phi=\pi$, leading to the fermion-parity anomaly as the short topological Josephson junction does. The behaviour of $I_{e 0}$ as a function of $\phi$ and $N_{g}$ is shown in Fig. 
2. Although our results hold only when $N_{g} \approx N_{0}+1 / 2$, the DC Josephson current already shows the oscillatory behaviour due to the charging energy.

\section{C. quenched dynamics}

Now we consider an abrupt change of the flux, i.e. $\phi=$ $\phi_{0}$ and $\phi_{0}+\phi_{1}$ for $t<0$ and $t>0$, respectively. This amounts to giving an initial state, say $|\Psi(0)\rangle=\left|+; \phi_{0}\right\rangle$, which is not the eigenstates of $H\left(\phi_{0}+\phi_{1}\right)$. We would like to calculate the resulting Josephson current at $t>0$.

The current operator $\hat{I}$ is defined as

$$
\hat{I} \equiv-2 e \frac{\partial H}{\partial \phi} .
$$

With the choice of the initial state given above, the Josephson current at $T=0$ for the sector with even fermion parity exhibits the behavior of Rabi oscillation:

$$
I_{e}(t)=I_{0} \cos \left(\Omega_{e} t+\alpha\right),
$$

where $I_{0}=e\left|\left(\Delta_{l}-\Delta_{r}\right) c_{+}^{*} c_{-}\right|$is the current amplitude, $\Omega_{e}=E_{+}-E_{-}=\sqrt{\epsilon_{d}^{2}+4\left|\Delta_{l}+\Delta_{r}\right|^{2}}$ is the Rabi frequency, $\alpha \equiv \arg \left[\left(\Delta_{l}-\Delta_{r}\right) c_{+}^{*} c_{-}\right], c_{ \pm}=\left\langle \pm ; \phi \mid+; \phi_{0}\right\rangle$, and $\phi=\phi_{0}+\phi_{1}$. We notice that $I_{0}, \Omega_{e}$, and $\alpha$ are all periodic functions of $\phi$. Especially, the period for $\Omega_{e}$ is $4 \pi$, which is a signature of the zero-energy MBSs.

We would like to emphasize a few points on the above results. First of all, the Josephson current will still exhibit the Rabi oscillation even if the change of $\Phi_{B}$ has a finite duration $\tau$. The requirement is that $\tau \Omega_{e} \ll 1$. Next, by taking into account the dissipation that we have ignored, the current will eventually decay into its equilibrium value given by Eq. (9). However, the oscillatory behavior still exists before the equilibrium value is reached, and the frequency $\Omega_{e}$ can be measured. Finally, in the presence of quasiparticle poisoning, the states with even and odd fermion parity will both contribute to the Josephson current. Since the current operator $\hat{I}$ commutes with the fermion-parity operator, the contributions from the states with different fermion parity will not mix, i.e. no interference appearing in the Josephson current. Therefore, the resulting current still exhibits an oscillatory behavior with two Rabi frequencies $\Omega_{e}$ and $\Omega_{o}$, where $\Omega_{o}=\tilde{E}_{+}-\tilde{E}_{-}=\sqrt{\epsilon_{d}^{2}+4\left|\Delta_{l}-\Delta_{r}\right|^{2}}$. Both are $4 \pi$-periodic functions of $\phi$. In this sense, the dependence of the Rabi frequencies on $\phi$ is immune to quasiparticle poisoning.

\section{THE STRONG TUNNELING REGIME}

\section{A. The model}

Now we turn into the strong tunneling regime, i.e. $G_{l / r} \approx e^{2} / h$ or $\mathcal{T}_{l / r} \approx 1$, where $\mathcal{T}_{l(r)}$ denotes the transmission coefficient of the left (right) contact. In this case, the dot is connected to the SC electrodes through a narrow constriction. We assume that the width of the constriction at its center allows only a single transverse state below the Fermi level. Since the constriction is formed electrostatically, its boundary is smooth and do not scatter electrons ${ }^{29}$. Following the work of Furusaki and Matveev $\stackrel{30}{\underline{3}}$, the whole system at $\mathcal{T}_{l / r}=1$ amounts to a superconductor-normal-metalsuperconductor (SNS) junction with a finite charging energy. Hence, the Hamiltonian at $\mathcal{T}_{l / r}=1$ can be written as $H_{0}+H_{c}$, where at the energy scale much lower than the bulk Fermi energy, $H_{0}$ can be written as $\underline{31}$

$$
\begin{aligned}
H_{0}= & \frac{v_{F}}{2} \int_{-\infty}^{\infty} d x\left[\left(\partial_{x} \Theta\right)^{2}+\left(\partial_{x} \Phi\right)^{2}\right] \\
& +V_{p} \int_{-\infty}^{0} d x \cos \left[\sqrt{4 \pi} \Theta(x)+\chi_{l}\right] \\
& +V_{p} \int_{L}^{+\infty} d x \cos \left[\sqrt{4 \pi} \Theta(x)+\chi_{r}\right],
\end{aligned}
$$

with $V_{p}=2 \Delta_{0} \sin \left(k_{F} a_{0}\right) /\left(\pi a_{0}\right)$, and

$$
H_{c}=E_{c}\left(\hat{N}-N_{g}\right)^{2},
$$

is the charging energy, with the exact form of $\hat{N}$ specified later. In the above, $v_{F}$ is the Fermi velocity, $k_{F}$ is the Fermi momentum, $a_{0}$ is a short-distance cutoff, and $\Delta_{l(r)}=\Delta_{0} e^{i \chi_{l(r)}}$ denotes the $p$-wave pairing amplitude in the left (right) SC electrode with $\Delta_{0}>0$. Moreover, we take the center of the left contact and that of the right contact to be located at $x=0$ and $x=L$, respectively. The bosonic fields $\Phi$ and $\Theta$, which obey the commutation relation $[\Phi(x), \Theta(y)]=i \theta(y-x)$ with $\theta(x)$ being the Heaviside unit step function, are related to the right mover $\psi_{+}$and the left mover $\psi_{-}$in the normal region through the bosonization formula:

$$
\psi_{ \pm}=\frac{1}{\sqrt{2 \pi a_{0}}} \exp [ \pm i \sqrt{\pi}(\Phi \mp \Theta)] .
$$

This model holds when $E_{c} \gg v_{F} / L$, where $v_{F} / L$ is the average level spacing in the QD. When $\mathcal{T}_{l / r}$ are close to one, we have to add a term $H_{b s}$ to $H_{0}+H_{c}$ to describe the weak backscattering at the contacts, where

$$
\begin{aligned}
& H_{b s}=v_{F}\left[\left|r_{l}\right| \psi_{+}^{\dagger}(0) \psi_{-}(0)+\left|r_{r}\right| \psi_{+}^{\dagger}(L) \psi_{-}(L)+\text { H.c. }\right] \\
& =-\frac{v_{F}}{\pi a_{0}}\left\{\left|r_{l}\right| \sin [\sqrt{4 \pi} \Phi(0)]+\left|r_{r}\right| \sin [\sqrt{4 \pi} \Phi(L)]\right\} .(17)
\end{aligned}
$$

and $r_{l(r)}$ is reflection amplitude at the left (right) contact. We notice that $H_{0}$ [Eq. (15)] has been used in Ref. 31 to describe the topological SNS junction. In our case, the Hamiltonian consists of an additional term - the charging energy $H_{c}$. We shall see later that the presence of $H_{c}$ will enhance the effects of $H_{b s}$, by turning it from an irrelevant perturbation to a marginal one.

Without loss of generality, we may shift the value of $\Theta$ by $\Theta(x) \rightarrow \Theta(x)-\chi_{l} / \sqrt{4 \pi}$ such that $H_{0}$ can be written 
as

$$
\begin{aligned}
H_{0}= & \frac{v_{F}}{2} \int_{-\infty}^{\infty} d x\left[\left(\partial_{x} \Theta\right)^{2}+\left(\partial_{x} \Phi\right)^{2}\right] \\
& +V_{p} \int_{-\infty}^{0} d x \cos [\sqrt{4 \pi} \Theta(x)] \\
& +V_{p} \int_{L}^{+\infty} d x \cos [\sqrt{4 \pi} \Theta(x)-\phi] .
\end{aligned}
$$

At the energy scale much smaller than the SC gap $\Delta_{0}$, the pairing terms suppress the fluctuations of $\Theta(x)$ in the regions $x<0$ and $x>L$. Thus, for $E \ll \Delta_{0}$, one may integrate out the degrees of freedom in these regions, and the resulting $H_{0}$ becomes

$$
H_{0}=\frac{v_{F}}{2} \int_{0}^{L} d x\left[\left(\partial_{x} \Theta\right)^{2}+\left(\partial_{x} \Phi\right)^{2}\right],
$$

with the boundary conditions

$$
\Theta(0)=0, \quad \Theta(L)=\frac{\phi}{\sqrt{4 \pi}}-\sqrt{\pi} \hat{J} .
$$

In Eq. (20), $\hat{J}$ is a Hermitian operator with integer eigenvalues. The boundary conditions (20), in fact, describe the perfect Andreev reflections at $x=0, L$, which is the characteristic for the interface between the normal region and the TSC 33 .

Now we can write down the expression of the Hermitian operator $\hat{N}$, which is given by

$$
\hat{N}=\frac{1}{\sqrt{\pi}} \int_{0}^{L} d x \partial_{x} \Phi=\frac{\Phi(L)-\Phi(0)}{\sqrt{\pi}} .
$$

Hence, $H_{c}$ becomes

$$
H_{c}=\frac{E_{c}}{\pi}\left[\Phi(L)-\Phi(0)-\sqrt{\pi} N_{g}\right]^{2} .
$$

Our working Hamiltonian is $H=H_{0}+H_{c}+H_{b s}$ with $H_{0}, H_{c}$, and $H_{b s}$ given by Eqs. (19), (22), and (17), respectively. Moreover, we shall assume that $\left|r_{l / r}\right| \ll 1$.

The mode expansions of $\Phi(x)$ and $\Theta(x)$ consistent with the boundary conditions (20) are of the forms

$$
\begin{aligned}
& \Phi(x)=-\frac{\hat{q}}{\sqrt{\pi}}+\bar{\Phi}(x) \\
& \Theta(x)=\left(\frac{\phi}{\sqrt{4 \pi}}-\sqrt{\pi} \hat{J}\right) \frac{x}{L}+\bar{\Theta}(x),
\end{aligned}
$$

where the Hermitian operator $\hat{q}$ is conjugate to $\hat{J}$, i.e. $[\hat{q}, \hat{J}]=i$, and

$$
\begin{aligned}
& \bar{\Phi}(x)=\sum_{n=1}^{+\infty} \frac{i}{\sqrt{q_{n} L}} \cos \left(q_{n} x\right)\left(a_{n}-a_{n}^{\dagger}\right), \\
& \bar{\Theta}(x)=\sum_{n=1}^{+\infty} \frac{1}{\sqrt{q_{n} L}} \sin \left(q_{n} x\right)\left(a_{n}+a_{n}^{\dagger}\right),
\end{aligned}
$$

with $q_{n}=n \pi / L$. In the above, $a_{n}$ and $a_{n}^{\dagger}$ obey the canonical commutation relations. Moreover, both commute with $\hat{q}$ and $\hat{J}$. Inserting Eqs. (23) and (24) into Eq. (19) gives

$$
H_{0}=\sum_{n=1}^{+\infty} v_{F} q_{n} a_{n}^{\dagger} a_{n}+\frac{\pi v_{F}}{2 L}\left(\hat{J}-\frac{\phi}{2 \pi}\right)^{2} .
$$

The second term in Eq. 25) describes the dynamics of the zero modes, while the first term describes the excitations in the QD.

\section{B. The effective action of the zero modes}

From Eq. (25), we notice that $\phi$ appears only in the zero modes. Thus, to study the Josephson effect, we would like to integrate out the $\bar{\Phi}$ field to get an effective Hamiltonian for the zero modes. We do this by two steps. First of all, we notice that $H_{c}$ and $H_{b s}$ depend only on $\Phi(0)$ and $\Phi(L)$. This observation suggests us to integrate out $\bar{\Phi}(x)$ with $x \neq 0, L$ to get an effective theory for $\phi_{l}=\bar{\Phi}(0)$ and $\phi_{r}=\bar{\Phi}(L)$.

To achieve this goal, we turn into the path-integral formulation. Since the action is Gaussian in $\bar{\Phi}(x)$ with $x \neq 0, L$, the integral can be done exactly and the resulting effective action for $\phi_{l}$ and $\phi_{r}$ is given by

$$
\begin{aligned}
S_{e f f}= & \frac{1}{2} \int_{0}^{\beta} d \tau_{1} d \tau_{2} \phi_{I}\left(\tau_{1}\right) \Delta_{I}^{-1}\left(\tau_{1}-\tau_{2}\right) \phi_{I}\left(\tau_{2}\right) \\
& +\frac{1}{2} \int_{0}^{\beta} d \tau_{1} d \tau_{2} \phi_{C}\left(\tau_{1}\right) \Delta_{C}^{-1}\left(\tau_{1}-\tau_{2}\right) \phi_{C}\left(\tau_{2}\right) \\
& -\lambda_{l} \int_{0}^{\beta} d \tau \sin \left[\sqrt{2 \pi}\left(\phi_{I}-\phi_{C}\right)-2 q\right] \\
& -\lambda_{r} \int_{0}^{\beta} d \tau \sin \left[\sqrt{2 \pi}\left(\phi_{I}+\phi_{C}\right)-2 q\right] \\
& +\frac{2 E_{c}}{\pi} \int_{0}^{\beta} d \tau\left(\phi_{C}-\sqrt{\frac{\pi}{2}} N_{g}\right)^{2}
\end{aligned}
$$

where $\phi_{l}=\left(\phi_{I}-\phi_{C}\right) / \sqrt{2}, \phi_{r}=\left(\phi_{I}+\phi_{C}\right) / \sqrt{2}, \lambda_{l / r}=$ $v_{F}\left|r_{l / r}\right| /\left(\pi a_{0}\right)$, and

$$
\begin{aligned}
\Delta_{I}(\tau) & =D(\tau ; 0,0)+D(\tau ; 0, L), \\
\Delta_{C}(\tau) & =D(\tau ; 0,0)-D(\tau ; 0, L) .
\end{aligned}
$$

In the above,

$$
D\left(\tau ; x_{1}, x_{2}\right) \equiv\left\langle\mathcal{T}_{\tau}\left\{\bar{\Phi}\left(\tau_{1}, x_{1}\right) \bar{\Phi}\left(\tau_{2}, x_{2}\right)\right\}\right\rangle_{0},
$$

is the free propagator of $\bar{\Phi}$, where $\tau \equiv \tau_{1}-\tau_{2}$ and $\mathcal{T}_{\tau}$ denotes time ordering along the imaginary-time axis. For the temperature $T \gg v_{F} / L$, we have

$$
\tilde{\Delta}_{I}\left(i \omega_{l}\right) \approx \frac{1}{\left|\omega_{l}\right|}, \quad \tilde{\Delta}_{C}\left(i \omega_{l}\right) \approx \frac{1}{\left|\omega_{l}\right|}
$$

where $\omega_{l}=2 l \pi T$ with $l$ being integer and $\tilde{A}\left(i \omega_{l}\right)$ is the Fourier transform of $A(\tau)$. 
At the energy scale much smaller than $E_{c}$, the charging energy $H_{c}$ suppresses the fluctuations of $\phi_{C}$, by setting its average value to be $\left\langle\phi_{C}\right\rangle=\sqrt{\pi / 2} N_{g}$. Thus, at energy scales much smaller than $E_{c}$, one may integrate out the fluctuations of $\phi_{C}$ by replacing the scattering terms in $S_{\text {eff }}[$ Eq. (26)] with its values averaged over the Gaussian fluctuations of $\phi_{C}$, yielding the effective action for $\phi_{I}$

$$
\begin{aligned}
\tilde{S}= & \frac{1}{2} \int_{0}^{\beta} d \tau_{1} d \tau_{2} \phi_{I}\left(\tau_{1}\right) \Delta_{I}^{-1}\left(\tau_{1}-\tau_{2}\right) \phi_{I}\left(\tau_{2}\right) \\
& +\int_{0}^{\beta} d \tau\left[\tilde{\lambda} e^{i\left(\sqrt{2 \pi} \phi_{I}-2 q\right)}+\text { H.c. }\right],
\end{aligned}
$$

where $\gamma=0.5772156649 \cdots$ is the Euler's constant and

$$
\tilde{\lambda}=\frac{2 i e^{\gamma} E_{c}}{\pi^{2}}\left(\left|r_{l}\right| e^{-i \pi N_{g}}+\left|r_{r}\right| e^{i \pi N_{g}}\right) .
$$

This is, in fact, a perturbative expansion in $\lambda_{l / r}$, which is justified because $\left|r_{l / r}\right| \ll 1$ and the fluctuations of $\phi_{C}$ around its expectation value are gapped.

Now we are in a position to integrate out $\phi_{I}$. We notice that $\tilde{\Delta}_{I}^{-1}\left(i \omega_{l}\right)=\left|\omega_{l}\right|$, which implies that the scaling dimension of the $\tilde{\lambda}$ term is 1 . That is, it is a marginal perturbation. (In the absence of the charging energy $H_{c}$, the scaling dimension of $H_{b s}$ is 2 so that it is an irrelevant perturbation $\underline{33}$. In this sense, the large charging energy enhances the effects of $H_{b s}$ on low-energy physics by freezing parts of the charge fluctuations.) Since $\left|r_{l / r}\right| \ll 1$, we may integrate out $\phi_{I}$ by the perturbative expansion in $\tilde{\lambda}$, yielding the effective action for the zero modes:

$$
\mathcal{I}=\mathcal{I}_{0}+\sum_{n=1}^{+\infty} \delta \mathcal{I}_{n}
$$

where $\delta \mathcal{I}_{n}=O\left(|\tilde{\lambda}|^{2 n}\right)$ and

$$
\mathcal{I}_{0}=\int_{0}^{\beta} d \tau\left[J\left(-i \partial_{\tau} q\right)+\frac{\pi v_{F}}{2 L}\left(J-\frac{\phi}{2 \pi}\right)^{2}\right] .
$$

The partition function of the TSC-QD-TSC junction is then given by

$$
Z=\int D[q] \sum_{J(\tau)=-\infty}^{+\infty} e^{-\mathcal{I}}
$$

Since the eigenvalues of $\hat{J}$ take integer values, $q$ must be an angular variable. Without loss of generality, we take $0 \leq q<2 \pi$ and the periodic boundary condition, i.e. $q(\beta)=q(0)$ in Eq. (30).

It is convenient to calculate the free energy in terms of a dual representation of Eq. (30). To achieve this goal, we first perform the summation over $J$. By using the Hubbard-Stratonovich transformation, we find that

$$
\sum_{J(\tau)=-\infty}^{+\infty} e^{-\mathcal{I}_{0}}=\int D[\rho] e^{-\frac{L}{2 \pi v_{F}} \rho^{2}+i \frac{\phi}{2 \pi} \rho} Q[\rho, q],
$$

where

$$
\begin{aligned}
& Q[\rho, q]=\sum_{J(\tau)=-\infty}^{+\infty} \exp \left[i \int_{0}^{\beta} d \tau J\left(\partial_{\tau} q-\rho\right)\right] \\
& =\sum_{J_{j}=-\infty}^{+\infty} \exp \left[i \lim _{N \rightarrow+\infty} \sum_{j=0}^{N-1} J_{j}\left(q_{j+1}-b_{j+1}-q_{j}+b_{j}\right)\right] \\
& =\lim _{N \rightarrow+\infty} \prod_{j=0}^{N-1} \sum_{J_{j}=-\infty}^{+\infty} e^{i J_{j}\left(q_{j+1}-b_{j+1}-q_{j}+b_{j}\right)},
\end{aligned}
$$

with $A_{j}=A\left(\tau_{j}\right), \Delta \tau=\beta / N$, and $\tau_{j}=j \Delta \tau$. Moreover, we have written $\rho=\partial_{\tau} b$. By using the Poisson summation formula

$$
\sum_{n=-\infty}^{\infty} \delta(x-2 \pi n)=\frac{1}{2 \pi} \sum_{m=-\infty}^{\infty} e^{i m x} .
$$

we get

$Q[\rho, q]=\lim _{N \rightarrow+\infty} \prod_{j=0}^{N-1} \sum_{l=-\infty}^{+\infty} \delta\left(\frac{q_{j+1}-b_{j+1}-q_{j}+b_{j}}{2 \pi}-l\right)$.

We see that $Q[\rho, q]$ provides each time slice a $\delta$-function which imposes the constraints $b_{j+1}-b_{j}=q_{j+1}-q_{j}-$ $2 \pi l_{j}$. The integration over $b$ in the presence of these local constraints leads to a global one $b(\beta)-b(0)=2 m \pi$ where $m=-\sum_{j=1}^{N-1} l_{j}$ is an integer. Since $\rho(\beta)=\rho(0)$, this is possible only when $b(\tau)=q(\tau)+2 m \pi \tau / \beta$. Because $b$ appears in the action only in the guise of $\partial_{\tau} b$, we may rewrite $Q[\rho, q]$ as

$$
Q[\rho, q]=\sum_{m=-\infty}^{+\infty} \delta\left[b(\tau)-q(\tau)-\frac{2 m \pi \tau}{\beta}\right] .
$$

Now we are in a position to integrate out $b$, and the partition function $Z$ becomes

$$
Z=\sum_{m=-\infty}^{+\infty} \int_{q(\beta)-q(0)=2 m \pi} D[q] e^{-\tilde{I}}
$$

where

$$
\tilde{I}=\int_{0}^{\beta} d \tau\left[\frac{L}{2 \pi v_{F}}\left(\partial_{\tau} q\right)^{2}-i \frac{\phi}{2 \pi} \partial_{\tau} q\right]+\sum_{n=1}^{+\infty} \delta \mathcal{I}_{n} .
$$

The effects of the term $2 m \pi \tau / \beta$ have been taken into account by changing the boundary condition for $q(\tau)$ from the periodic one to $q(\beta)-q(0)=2 m \pi$. Equation (31) is the desired dual representation of Eq. (30). We shall use both in the following.

\section{The DC Josephson current}

Now we are able to compute the DC Josephson current. When $\tilde{\lambda}=0$ or $\mathcal{T}_{l / r}=1$, the action $\tilde{I}$ is nothing but 
the one for a particle, of mass $L /\left(\pi v_{F}\right)$ and unit charge, moving on a unit circle thread by a flux $\phi$, provided that we treat $q$ as the azimuthal angle. In this sense, the integer $m$ is the winding number. Hence the partition function at $\mathcal{T}_{l / r}=1$ can be written as

$$
Z_{0}(\phi)=\sum_{J=-\infty}^{+\infty} e^{-\beta \epsilon_{J}}
$$

leading to the free energy $F_{0}=-T \ln Z_{0}$, where $\epsilon_{J}=$ $\frac{\pi v_{F}}{2 L}[J-\phi /(2 \pi)]^{2}$ is the energy of the zero mode with the quantum number $J$. The resulting DC Josephson current is of the form

$$
I^{(0)}(\phi)=\frac{e v_{F}}{L} \sum_{J=-\infty}^{+\infty}\left(J-\frac{\phi}{2 \pi}\right) P_{J},
$$

where $P_{J}=\frac{1}{Z_{0}} e^{-\beta \epsilon_{J}}$ is the probability of the zero mode at the eigenstate $|J\rangle$. We notice that the whole spectrum is $2 \pi$-periodic in $\phi$, and thus $I^{(0)}$ is a $2 \pi$-periodic function of $\phi$. Moreover, $I^{(0)}(-\phi)=-I^{(0)}(\phi)$, which implies that $I^{(0)}(0)=0$. The eigenvalue of $\hat{J}$ is the difference between the numbers of the right- and the left-movers. Hence, even and odd values of $J$ correspond to different fermion parities. By taking into account the conservation of fermion parity, the allowed values of $J$ become either odd or even integers. Consequently, $I^{(0)}$ turns into a $4 \pi$-periodic function of $\phi$, a characteristic of topological Josephson junctions.

To proceed, it is more convenient to use the dual representation of $Z_{0}$ [Eq. (31)]. By writing $q(\tau)=$ $\bar{q}(\tau)+2 m \pi \tau / \beta$ with $\bar{q}(\beta)=\bar{q}(0)$ and then performing the integration over $\bar{q}$, we obtain $Z_{0}$

$$
Z_{0}(\phi)=\sqrt{\frac{2 L}{v_{F} \beta}} \sum_{m=-\infty}^{+\infty} e^{i m \phi-\frac{L}{2 \pi v_{F} \beta}(2 m \pi)^{2}} .
$$

Since $T \gg v_{F} / L$, the dominant contributions arise from the terms with $m=0$ and $m= \pm 1$. Hence, we may approximate $Z_{0}$ as

$$
Z_{0}(\phi) \approx \sqrt{\frac{2 L}{v_{F} \beta}}\left(1+2 e^{-\frac{2 \pi L}{v_{F} \beta}} \cos \phi\right) .
$$

Consequently, the DC Josephson current is given by

$$
I^{(0)}(\phi) \approx 4 e T e^{-\frac{2 \pi L}{v_{F} \beta}} \sin \phi
$$

which holds when $v_{F} / L \ll T \ll E_{c}$.

In the above calculation, we do not take into account the conservation of fermion parity so that $I^{(0)}$ is a $2 \pi$ periodic function of $\phi$. The constraint from the fermionparity conservation can be included by assuming that $J$ must be even (or odd) integers. With the similar procedure, we find that

$$
I_{e}^{(0)}(\phi) \approx 2 e T e^{-\frac{\pi L}{2 v_{F}^{\beta}}} \sin (\phi / 2)=-I_{o}^{(0)}(\phi) .
$$

We see that $I_{e}^{(0)}$ and $I_{o}^{(0)}$ have opposite signs at given $T$ and $\phi$.

When $\tilde{\lambda} \neq 0$, small reflection amplitudes at the contacts will modify the DC Josephson current. We shall calculate the corrections to $\mathcal{I}^{(0)}$ by the perturbative expansion in $|\tilde{\lambda}|^{2}$. The leading contribution arises from $\delta \mathcal{I}_{1}$ which takes the form

$\delta \mathcal{I}_{1}=-|\tilde{\lambda}|^{2} \int_{0}^{\beta} d \tau_{1} d \tau_{2} V\left(\tau_{1}-\tau_{2}\right) \cos \left\{2\left[q\left(\tau_{1}\right)-q\left(\tau_{2}\right)\right]\right\}$,

where

$$
\begin{aligned}
V(\tau) & =\left\langle e^{i \sqrt{2 \pi} \phi_{I}(\tau)} e^{-i \sqrt{2 \pi} \phi_{I}(0)}\right\rangle \\
& =\exp \left\{2 \pi\left[\Delta_{I}(\tau)-\Delta_{I}(0)\right]\right\} .
\end{aligned}
$$

Using the Baker-Hausdorff formula, one may verify that

$$
\left[\hat{J}, e^{i \alpha \hat{q}}\right]=\alpha e^{i \alpha \hat{q}}
$$

This equation indicates that the operator $e^{i \alpha \hat{q}}$ shifts the eigenvalue of $\hat{J}$ by $\alpha$. Thus, the effects of $\delta \mathcal{I}_{1}$ is to shift the eigenvalue of $\hat{J}$ by \pm 2 . In general, the terms in $\delta \mathcal{I}_{n}$ will shift the eigenvalue of $\hat{J}$ by $\pm 2 n$. As a result, all the terms $\delta \mathcal{I}_{n}$ do not change the fermion parity.

In general, a perturbative expansion of the free energy $F$ can be written as

$$
F=F_{0}+\sum_{n=1}^{+\infty} F_{n}
$$

where $F_{n}=O\left(|\tilde{\lambda}|^{2 n}\right)$. In particular, we have

$$
F_{1}=T\left\langle\delta \mathcal{I}_{1}\right\rangle_{0}=-T|\tilde{\lambda}|^{2} \int_{0}^{\beta} d \tau_{1} d \tau_{2} V(\tau) C(\tau ; \phi)
$$

where $\langle\cdots\rangle_{0}$ denotes the average with respect to $Z_{0}, \tau=$ $\tau_{1}-\tau_{2}$, and

$$
C(\tau ; \phi)=\left\langle\cos \left\{2\left[\hat{q}\left(\tau_{1}\right)-\hat{q}\left(\tau_{2}\right)\right]\right\}\right\rangle_{0} .
$$

When $T \gg v_{F} / L, C(\tau ; \phi)$ can be approximated as

$$
\begin{aligned}
C(\tau ; \phi) \approx & {\left[1-4 e^{-\frac{2 \pi L}{v_{F} \beta}} \cos \phi \sin ^{2}\left(\frac{2 \pi \tau}{\beta}\right)\right] } \\
& \times \exp \left[-\frac{2 \pi v_{F}}{\beta L} \tau(\beta-\tau)\right]
\end{aligned}
$$

for $0 \leq \tau \leq \beta$.

Collecting the above results, $F_{1}$ is given by

$$
\begin{aligned}
F_{1} \approx & -2 T|\tilde{\lambda}|^{2} \int_{0}^{\beta} d \tau \tau V(\tau) \exp \left[-\frac{2 \pi v_{F}}{\beta L} \tau(\beta-\tau)\right] \\
\times & {\left[1-4 e^{-\frac{2 \pi L}{v_{F} \beta}} \cos \phi \sin ^{2}\left(\frac{2 \pi \tau}{\beta}\right)\right] }
\end{aligned}
$$

when $v_{F} / L \ll T \ll E_{c}$, which leads to the leading correction to the DC Josephson current

$$
I^{(1)}=-\frac{16 \pi^{2} e T|\tilde{\lambda}|^{2}}{E_{c}^{2}} e^{-\frac{2 \pi L}{v_{F}^{\beta}}} \sin \phi .
$$



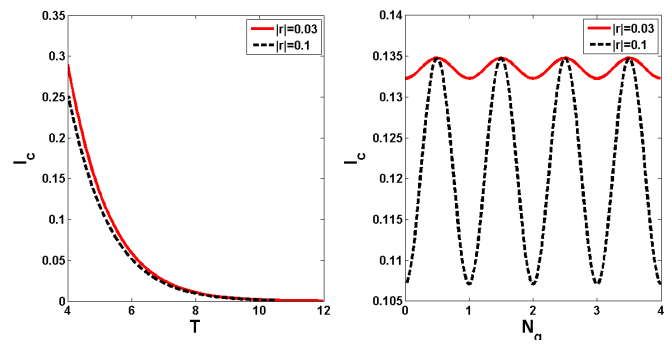

FIG. 3: (Color online) The critical current $I_{c}$ [Eq. (40)] for a TSC-QD-TSC junction in the strong-tunneling regime. We set $\left|r_{l}\right|=\left|r_{r}\right|=|r|$ and measure $I_{c}$ in units of $e v_{F} /(2 \pi L)$. Left: $I_{c}$ versus the temperature $T$ (in units of $v_{F} /(2 \pi L)$ ) at $N_{g}=0.2$ for $|r|=0.03$ (solid line) and $|r|=0.1$ (dashed line). Right: $I_{c}$ versus $N_{g}$ at $T=5 v_{F} /(2 \pi L)$ for $|r|=0.03$ (solid line) and $|r|=0.1$ (dashed line).

As a result, we find that for $v_{F} / L \ll T \ll E_{c}$, the DC Josephson current is of the form

$$
I=I_{2 \pi} \sin \phi,
$$

where the critical current $I_{2 \pi}$ is given by

$$
I_{2 \pi}=4 e T e^{-\frac{2 \pi L}{v_{F} \beta}}\left(1-\frac{4 \pi^{2}|\tilde{\lambda}|^{2}}{E_{c}^{2}}\right)
$$

to the leading order in $|\tilde{\lambda}|^{2}$. We see that the small reflection amplitudes at the contacts do not change the current-phase relation. They manifests themselves in the critical current in two ways. (i) First of all, the small reflection amplitudes at the contacts diminish the critical current. (ii) Next, the current acquires the dependence on the gate voltage through the reflection amplitudes because

$$
\frac{4 \pi^{2}|\tilde{\lambda}|^{2}}{E_{c}^{2}}=\left(\frac{4 e^{\gamma}}{\pi}\right)^{2}\left[\left|r_{l}\right|^{2}+\left|r_{r}\right|^{2}+2\left|r_{l}\right|\left|r_{r}\right| \cos \left(2 \pi N_{g}\right)\right] .
$$

Hence, at given temperature, the critical current reaches the maximal values for half-integer $N_{g}$ and the minimal values for integer $N_{g}$. The behaviours of $I_{2 \pi}$ versus $T$ and $N_{g}$ are shown in Figs. 3. By comparing with the results in the weak-tunneling regime, we conclude that the current-phase relation for the TSC-QD-TSC junction can be changed by varying the values of $\mathcal{T}_{l / r}$, which can be achieved by adjusting the applied gate voltages which form the constrictions.

We notice that $I^{(1)}$ has the same temperature dependence as $I^{(0)}$. This reflects the fact that $H_{b s}$ is a marginal perturbation, which follows from the large charging energy. If there were no charging energy, $H_{b s}$ would be an irrelevant perturbation such that an additional factor $T$ would appear in $I^{(1)}$. Consequently, the effects of $H_{b s}$ would be suppressed at low temperatures 33 . Our analysis indicates that the effects of reflections at the contacts are enhanced in the presence of a large charging energy.
In the above calculation, we do not take into account the conservation of fermion parity. For fixed fermion parity, the only change in $F_{1}$ lies in the function $C(\tau ; \phi)$, and we obtain

$$
I_{e}=I_{4 \pi} \sin (\phi / 2)=-I_{o},
$$

where

$$
I_{4 \pi}=2 e T e^{-\frac{\pi L}{2 v_{F} \beta}}\left(1-\frac{4 \pi^{2}|\tilde{\lambda}|^{2}}{E_{c}^{2}}\right),
$$

is the critical current with fixed fermion parity. We see that $I_{e}$ and $I_{o}$ have opposite signs at given $T, \phi$, and $N_{g}$. Moreover, the ratio $I_{4 \pi} / I_{2 \pi}$ depends on the temperature $T$ in a nontrivial way, but is independent of the gate voltage.

\section{CONCLUSIONS AND DISCUSSIONS}

We study the DC Josephson effect in a TSC-QD-TSC junction. In the weak-tunneling regime, the Andreev spectrum becomes simple near the degenerate point of the QD. This simple structure should appear as sharp peaks in the spectral function of the dot, which could be observed by established experimental techniques. We also consider the abrupt change of the flux, which results in a Rabi oscillation of the Josephson current with frequencies being $4 \pi$-periodic functions of $\phi$. We indicate that this feature is immune to the quasiparticle poisoning. Far away from the degenerate point, the proliferation of Andreev levels will complicate the behavior of the Josephson current. Therefore, we do not consider such a situation. We notice that the use of the Rabi oscillation to extract the signature of the MBSs has been proposed in a different setup ${ }^{34}$. In that case, the QD is side-coupled to one end of the TSC in a Josephson junction, and it is the electron occupation of the dot which exhibits the Rabi oscillation due to the DC Josephson current.

In the strong-tunneling regime, we model the dot and the contacts as a one-dimensional electron liquid, following the work on the charge transport through an open $\mathrm{QD}^{30}$. Using the bosonization, we may calculate the free energy and the DC Josephson current in a perturbative expansion in the reflection amplitudes. The resulting current-phase relation is of the form $I_{c} \sin (\phi / 2)$, identical to the short Josephson junction. The difference lies at the critical current $I_{c}$, which acquires a nontrivial dependence on the temperature and the gate voltage. In the absence of the backscattering terms, the critical current we obtained is identical to the one for the long topological SNS junction in the temperature range $T \gg v_{F} / L \underline{ }$.

In the absence of the charging energy $H_{c}$, the lowenergy effective Hamiltonian is identical to the one for the long topological SNS junction ${ }^{31,32}$. The role of the charging energy is to enhance the effects of the backscattering at the contacts on low-energy properties. This has been shown in the study of the charge transport through 
the open $\mathrm{QD}^{30}$. There, the backscattering terms become relevant perturbations and a non-perturbative method is warranted. In the present case, thanks to the superconductivity in the bulk, the backscattering terms turn into a marginal perturbation, and a perturbative expansion works.

In the weak tunneling regime, we only consider the case near the degenerate point of the QD so that only two levels of the dot are involved. It is interesting to study how the Andreev spectrum and the resulting current-phase relation evolve upon moving far away from the degenerate point. On the other hand, our results in the strong tunneling regime hold only for $T \gg v_{F} / L$. Thus, the DC Josephson effect at the temperature range $T \approx v_{F} / L$ requires further study. Finally, as our analysis suggests, the current-phase relation of the TSC-QD-TSC junction depends on the transmission coefficients $\mathcal{T}_{l / r}$ of the contacts. Since the control of the critical current is important for the basic elements in large-scale integrated SC circuits 36 , the dependence of the current-phase relation on $\mathcal{T}_{l / r}$ is deserved to investigate.

\section{Acknowledgments}

The work of Y.-W. Lee is supported by the National Science Council of Taiwan under Grant No. NSC 1022112-M-029-002-MY3.
* Electronic address: yllee@cc.ncue.edu.tw

$\dagger$ Electronic address: ywlee@thu.edu.tw

1 J. Alicea, Rep. Prog. Phys. 75, 076501 (2012).

2 M. Leijnse and K. Flensberg, Semicond. Sci. Technol. 27, 124003 (2012).

3 C.W.J. Beenakker, Annu. Rev. Condens. Matter Phys. 4, 113 (2013).

${ }^{4}$ C.W.J. Beenakker, Rev. Mod. Phys. 87, 1037 (2015).

${ }^{5}$ D.A. Ivanov, Phys. Rev. Lett. 86, 268 (2001).

6 A.Y. Kitaev, Phys. Usp. 44, 131 (2001).

7 C. Nayak, S.H. Simon, A. Stern, M. Freedman, and S. Das Sarma, Rev. Mod. Phys. 80, 1083 (2008).

8 J. Alicea, Y. Oreg, G. Refael, F. von Oppen, and M.P.A. Fisher, Nat. Phys. 7, 412 (2011).

${ }^{9}$ V. Mourik, K. Zuo, S.M. Frolov, S.R. Plissard, E.P.A.M. Bakkers, and L.P. Kouwenhoven, Science 336, 1003 (2012).

10 A. Das, Y. Ronen, Y. Most, Y. Oreg, M. Heiblum, and H. Shtrikman, Nat. Phys. 8, 887 (2012).

11 M.T. Deng, C.L. Yu, G.Y. Huang, M. Larsson, P. Caroff, and H.Q. Xu, Nano Lett. 126414 (2012).

12 H.O.H. Churchill, V. Fatemi, K. Grove-Rasmussen, M.T. Deng, P. Caroff, H.Q. Xu, and C.M. Marscus, Phys. Rev. B 87, 241401 (2013).

13 L. Fu and C.L. Kane, Phys. Rev. B 79, 161408(R) (2009).

14 R.M. Lutchyn, J.D. Sau, and S. Das Sarma, Phys. Rev. Lett. 105, 077001 (2010).

15 K.T. Law and P.A. Lee, Phys. Rev. B 84, 081304(R) (2011).

16 D. Sticlet, C. Bena, and P. Simon, Phys. Rev. B 87, 104509 (2013).

17 F. Pientka, A. Romito, M. Duckheim, Y. Oreg, and F. von Oppen, New J. Phys. 15, 025001 (2013).

18 M. Houzet, J.S. Meyer, D.M. Badiane, and L.I. Glazman, Phys. Rev. Lett. 111, 046401 (2013).

19 B. van Heck, F. Hassler, A.R. Akhmerov, and C.W.J. Beenakker, Phys. Rev. B 84, 180502(R) (2011).
20 B. Tarasinski, D. Chevallier, J.A. Hutasoit, B. Baxevanis, and C.W.J. Beenakker, Phys. Rev. B 92, 144306 (2015).

21 K. Wölms and K. Flensberg, Phys. Rev. B 92, 165428 (2015).

22 M. Leijnse and K. Flensberg, Phys. Rev. B 84, 140501(R) (2011).

23 A. Golub, I. Kuzmenko, and Y. Avishai, Phys. Rev. Lett. 107, 176802 (2011).

24 M. Lee, J.S. Lim, and R. Lopez, Phys. Rev. B 87, 241402(R) (2013).

25 M. Cheng, M. Becker, B. Bauer, and R.M. Lutchyn, Phys. Rev. X 4, 031051 (2014).

26 M. Governale, M.G. Pala, and J. Konig, Phys. Rev. B 77, 134513 (2008).

27 J. Sköldberg, T. Löfwander, V.S. Shumeiko, and M. Fogelström, Phys. Rev. Lett. 101, 087702 (2008); P. Recher, Y.V. Nazarov, and L.P. Kouwenhoven, ibid. 104, 156802 (2010).

28 L. Fu, Phys. Rev. Lett. 104, 056402 (2010).

29 L.I. Glazmann, G.B. Lesovik, D.E. Khmel'nitskii, and R.I. Shekhter, JETP Lett. 48, 238 (1991).

30 A. Furusaki and K.A. Matveev, Phys. Rev. B 52, 16676 (1995).

31 M. Cheng and R. Lutchyn, Phys. Rev. B 92, 134516 (2015).

32 F. Crépin and B. Trauzettel, Phys. Rev. Lett. 112, 077002 (2014).

33 L. Fidkowski, J. Alicea, N.H. Lindner, R.M. Lutchyn, and M.P.A. Fisher, Phys. Rev. B 85, 245121 (2012).

34 Z. Wang, Q.F. Liang, D.X. Yao, and X. Hu, Sci. Rep. 5, 11686 (2015).

35 C.W.J. Beenakker, D.I. Pikulin, T. Hyart, H. Schomerus, and J.P. Dahlhaus, Phys. Rev. Lett. 110, 017003 (2013).

36 A.A. Golubov, M.Yu. Kupriyanov, and E. Ll'ichev, Rev. Mod. Phys. 76, 411 (2004). 